\title{
On Elliptic Operator Theory
}

\author{
Abdalla, Salih Abdalla ${ }^{1}$ \\ ${ }^{1}$ Department of Mathematics, University of El Imam El Mahdi, Sudan \\ Correspondence: Abdalla, Salih Abdalla, Department of Mathematics, University of El Imam El Mahdi, Sudan. \\ E-mail: s33@gmx.co.uk
}

Received: May 30, 2016 Accepted: June 10, 2016 Online Published: November 12, 2017

doi:10.5539/jmr.v9n6p144 URL: https://doi.org/10.5539/jmr.v9n6p144

\begin{abstract}
The theory talks about partial differentiation equations. For the this reason, elliptic operators take the portion of differential operators in general Laplace operators. The operators have clear definitions through the rule that the coefficients of the highest order derivatives are positives. The above aspects imply that crucial properties, which are the main symbols, are fixed. On the other hand, they can be equivalent, meaning that they have no real characteristic directions. To expound on the above, elliptic operators are representatives of the potential theory. The reason behind this is that they often appear in the field of electrostatics and continuum mechanics. Elliptic regularity shows that their solutions tend to be smooth functions, which means that it applies if the coefficients are smooth operators. Additionally, firm state resolutions to hyperbolic and parabolic equations apply in general to solve elliptic equations. Partial differentiation equations are important mathematical formulas that apply in solving mathematical problems. The theory plays a critical role in explaining functioning of the elliptic operator as many find it a mathematical phenomenon in how efficient it is in solving problems in mathematics. The article talks about theorems and lemmas in the elliptic operator.
\end{abstract}

Keywords: Elliptic Operator, Theorems, lemmas, operators, equations, partial differentiation

\section{Introduction}

There have been topical mathematical advancements on several theories including the elliptic operator theory. The advancements extend to the functioning of theorems and lemmas and the actual application on mathematical contexts. Consequently, the developments in elliptic operator theory have led to the question of whether Desargues's conjecture is true or false in relation to rings (Kim, 2015). On the contrary, the mathematical construction of partial triangles was a milestone in the Galois Theory, which has a relation to the elliptic operator. The theory shows characterization of holomorphic systems. The holomorphic system used the formulae

$$
-\infty^{7} \sim \beta\left(\pi-\infty, \ldots, \infty^{4}\right)
$$

In addition, the theory explains proofs for harmonic functions. The information could have equation representations through Harmacks inequality applied to $-f$ for $r<1$.

Harnack's inequality applied $-f$

$$
-f(r x) \geq-\frac{1-r}{(1+r)^{n-1}} f(0)
$$

$r<1$. Hence

$$
\frac{f(x)-f(r x)}{1-r}=\frac{-f(r x)}{1-r} \geq-\frac{1}{(1+r)^{n-1}} f(0)>-\frac{f(0)}{2^{n-1}}>0
$$

The second order and pattern followed in the equation assume the elliptic operator formulae and its application in solving mathematical problems. There is a directional on the right side, which is a positive constant. From a close view, the formulas are related. Below is the elliptic operator formula:

$$
L u=a_{i j}(x) \frac{\partial^{2} u}{\partial x_{i} \partial x_{j}}+b_{i}(x) \frac{\partial u}{\partial x_{i}}+c(x) u, \quad x \in \Omega .
$$

The article discusses the theorems, lemmas, formulas and application of the elliptic operator theory. 


\section{Theorems of the Elliptic Operator Theory}

\subsection{Atiyah-Singer Index Theorem}

One of the prolific theorems is AtiyahCSinger index theorem. Michael Atiyah and Isadore Singer were the individuals who proved the theorem in the year 1963. The theorem has it that that for proper function of the elliptic differential operator to operate on a condensed manifold. To expound on the above the analytical index is equivalent to the topological index. The analytical talks about relations in the dimensions of the spaces and its relevant solutions. Topological index talks about similar topological data or information. The theorem shows the relation between the two aspects.

The mathematical problem related to elliptic differential operators was posed in 1960. An Israelite Mathematician posed the problem after noticing several related aspects to the problem. He realized that the index had a variance in terms of homotopy, which lead to looking for relevant formulas that one can apply to solve the problems in mathematics (Carl, and VyK, 2014). Its founders, Atiyah and singer announced the theorem in the year 1963, which gave the reason behind its name Atiyah-singer theorem. The proof of the theorem appears vibrantly in the book Palais of the year 1965.

The theorem expresses notations. The first notation is $X$. $X$ expresses a compact smooth manifold, which means it is a manifold without any boundary. The second pair of notations are $E$ and $F$. These are smooth vector bundles over the variable $X$. the next variable is $D$, Which is a representation of an elliptic differential operator from $E$ to $F$. The above means that in the local coordinates, the variable acts as a differential operator, taking smooth sections of $E$ to smooth sections of $F$. The above representation leads on to the symbols of differential operators. One should use $D$ as the differential operator on a Euclidean space of order $n$ in $k$ variables. Most of the times, analytical index has $D$ in its representation.

$$
x_{1}, \ldots, x_{k}
$$

then its symbol is the function of $2 \mathrm{k}$ variables

$$
x_{1}, \ldots, x_{k}, y_{1}, \ldots y_{k}
$$

\subsection{RiemannC Roch Theorem}

The theorem is one of the most vital and significant aspects in the field of mathematics. The application is widely used in solving complex analytical mathematical problems and algebraic geometry in general. A prolific example of complex mathematical analysis is computation of dimensions of space. Such problems shows prescriptions of zeros and allowed poles (Cappiello, and Fabio, 2016). Most of the problems in this theorem are in algebraic formats. Riemann proved the theory through the help of inequalities in the year 1875. Algebraic curves played a crucial role in ensuring that the theory became general and had higher- dimensional varieties that were beyond the normal dimensional representations.

The theory has several mathematical representations. To start with, it has $X$ as a representation symbol of topological space. $\mathbb{C}$ on the other hand represents complex numbers in the theorem. The theory operates with $\mathbb{C}$ to the surface of variable $X$. The theory works with the assumption that the surface $X$ is compact. A divisor represented by the symbol $D$ is a component of the free Abelian group on the surface points of a mathematical problem (Fabio, 2015). Homogeneously, a divisor is a fixed linear amalgamation of points of the surfaces that have integers and coefficients. In case of any meromorphic function in the problem, $f$ increases the value of the divisor, where its denoted $(f)$ defined as

$$
(f):=\Sigma_{z_{v} \in R(f)} S_{v} s_{v}
$$

Whereby, $R(f)$ represents the groups of all zeroes and poles of $f$, and $s_{v}$ in the problem is given by the equation:

$$
S_{v}:= \begin{cases}a & \text { if } z_{v} \text { is a zero of order } a \\ -a & \text { if } z_{v} \text { is a pole of order } a\end{cases}
$$

\section{Elliptic Operator Theory, Lemma}

One of the most prominent mathematical lemmas was Hopf lemma founded by Eberhand Hopf. He was expressing the function of real value in the domain of Euclidean space (Behrndta and Michelerb, 2014). The lemma strongly uses the statement for harmonic functions as its framework basis in its functioning. Assuming that $\Omega$ is a bounded domain in the representation of $\mathbb{R}^{n}$,which comprises of a smooth boundary. Letting $f$ be a real-valued function continuing at the end of $\Omega$ and harmonic on $\Omega$. If $x$ is the boundary point such that $f(x)>f(y)$ for all $\mathrm{y}$ in $\Omega$ appropriately close to $x$, then the 
(one-sided) directional derivative of $f$ in the direction of the external, pointing out and being normal to the boundary at $x$, which is strictly positive Candito, Carl, and Livrea, 2013). The harmonic functions apply Harnacks inequality concepts as its baseline proof of the lemma.

Below are examples of equations as an attempt to explain the lemma. To start with the general elliptic operator in the form of an equation representation:

$$
L u=a_{i j}(x) \frac{\partial^{2} u}{\partial x_{i} \partial x_{j}}+b_{i}(x) \frac{\partial u}{\partial x_{i}}+c(x) u, \quad x \in \Omega
$$

From the above equation, it moves one to open bounded subset equation to explain the concept of Hopf. The Extreme Principles show a solution of the equation $l u=0$ in $\Omega$.From the equation, it attains its maximum value on the closure, whereby at some point, they appear on the boundary.

The lemma uses a derivation from the outer normal derivative. The derivation bases on the fact of non-decreasing as an approach to solve problems. The lemma is important and significant as it strengthens observations one gets from looking at mathematical solutions applied in several related problems. The lemma proves that proves mild assumption in mathematics to be true through the equation below:

$$
\frac{\partial u}{\partial v}\left(x_{0}\right)>0
$$

\section{Ultra hyperbolic Equation}

The equation is vital in mathematics as it solves problems significantly. The partial deferential equation s famous for its scalar of $\mathrm{U}$ of $2 \mathrm{n}$ variables in $\mathrm{X}$. problems and their solutions in this field follow the equation below:

$$
\frac{\partial^{2} u}{\partial x_{1}^{2}}+\ldots+\frac{\partial^{2} u}{\partial x_{n}^{2}}-\frac{\partial^{2} u}{\partial y_{1}^{2}}-\ldots-\frac{\partial^{2} u}{\partial y_{n}^{2}}=0
$$

The problem is efficient as it solves problems in forms of quadratic equations. The equation has two variables in it. The two variables are represented by signs $(n, n)$. The equations are placed in a format that one can use in application of solving related mathematical problems. The variables in the equation are interchangeable.

Several mathematician scholars have studied the equation in various perspectives. On one side, the equation looks like the looks like the wave equation. The reason behind this is that there are resembling characteristics and features that resemble each other. Such reasons have contributed to advancements in the characteristics of the equation. A good example of equations that share its characteristics to Ultra hyperbolic equation is the John equation. In addition, the equation has drawn several scholars to the ways of its functioning. Walter Craig and Steven Weinstein are examples of scholars who recently appraised on the equation. This was in the year 2008, where they added more strength to the proof of the theory under nonlocal constraints. To expound on this, the initial value of the mathematical problem to be solved were kept in the form of data given and represented in a similar dimension. People also view the equation in the terms of symmetric spaces in the mathematical field (Bobkov and Yavdat, 2015). The studies additionally link the theory to elliptic operator theory as it expresses the elliptic deferential operators and their ways of functioning to bring forth desired solutions to mathematic problems. The equation gives aspects of content to the value function of value functions for harmonic functionalities.

\section{Conclusion}

In conclusion, the elliptic operator theory is a significant theory in the entire mathematical field. The reason behind this is that it comprises of significant lemmas and mathematical theories applied in a global context to solve problems. The theory gives vibrant expressions and illustrations on ways in which related theories and lemmas function and act as framework base in finding mathematical problems. One of the prominent theorems is AtiyahCSinger index theorem of 1963. The theorem was significant as it shows progression of the theorem and the way the theorem joined the world of mathematics. In addition, the theorem is true as it has a strong proof of its functioning. The theorist clearly explains the origin of the theory and its relation to elliptic operator theory. Another important theory is the Reimann Roch theory. The theory is vital as it applies in solving algebraic equations and complex analytical mathematical problems such as computation of space. Such functions are crucial in mathematics as give solutions to complex problems, which expresses the role of the theorem in elliptic operations in mathematics. The Hopf lemma is another crucial part in the elliptic operator theory. The lemma is important as it links harmonic functions together. The above reasons makes the lemma important as harmonic functions form the baseline for the framework of elliptic operations. Harnacks inequality apply in the lemma to explain functioning of the equations used in solving mathematical problems. Another important part in elliptic operations is the Ultra hyperbolic equation. The equation uses two variables and quadratic equations. The variables are interchangeable. Although, most of the scholars have viewed the equation in different perspectives, where some have strengthened the 
proofs in relation to the equation and its relation to elliptic operations. For the above reasons, the elliptic operator theory plays a key role in explaining and giving proofs to most of the equations applied in the field of mathematics.

\section{References}

Behrndta, J., \& Michelerb, T. (2014).Elliptic Differential Operators on Lipschitz Domains and Abstract Boundary Value Problems. Journal of Functional Analysis ,267(10),3657C3709.

Bobkov, V., \& Yavdat, I. (2015). Maximal Existence Domains of Positive Solutions for Two- Parametric Systems of Elliptic Equations.Complex Variables and Elliptic Equations:An International Journal ,61(5),587-607. https://doi.org/10.1080/17476933.2015.1107905

Candito, P., Carl, S., \& Livrea, R. (2013). Variational Versus Pseudomonotone Operator Approach in Parameter-Dependent Nonlinear Elliptic Problems.Dynamic Systems and Applications,22,397-410.

Cappiello, M., \& Fabio, N. (2016) Sharp Decay Estimates and Smoothness for Solutions to Nonlocal Semi linear Equations.In: Discrete and Continuous Dynamical Systems C Series A,36(4),1869-1880.

Carl, S., \& Vy K. Le, (2014). Multi-Valued Parabolic Variational Inequalities and Related Variational-Hemivariational Inequalities.Advanced Nonlinear Studies ,14(1),603-631. https://doi.org/10.1515/ans-2014-0307

Fabio, N. (2015). On The Time Slicing Approximation Of Feynman Path Integrals For Non-Smooth.Journal D'analyse Mathematiques , arXiv:1506.01275. Potentials.http://arxiv.org/pdf/1506.01275v1.pdf

Kim, S. (2015). Ellipsoidal Microhydrodynamics without Elliptic Integrals and How to Get There Using Linear Operator Theory: A Note on Weighted Inner, School of Chemical Engineering, Purdue University.Ind. Eng. Chem. Res. 54(42),10549 C10551.

\section{Copyrights}

Copyright for this article is retained by the author(s), with first publication rights granted to the journal.

This is an open-access article distributed under the terms and conditions of the Creative Commons Attribution license (http://creativecommons.org/licenses/by/4.0/). 